\title{
Cluster size diversity, percolation, and complex systems
}

\author{
I. R. Tsang and I. J. Tsang \\ VisionLab-Department of Physics, University of Antwerp-RUCA, Groenenborgerlaan 171, Antwerp B-2020, Belgium
}

(Received 23 February 1999; revised manuscript received 20 May 1999)

\begin{abstract}
Diversity of cluster size has been used as a measurement of complexity in several systems that generate a statistical distribution of clusters. Using Monte Carlo simulations, we present a statistical analysis of the cluster size diversity and the number of clusters generated on randomly occupied lattices for the Euclidean dimensions 1 to 6. A tuning effect for diversity of cluster size and critical probabilities associated with the maximum diversity and the maximum number of clusters are reported. The probability of maximum diversity is related to the percolation threshold and several scaling relations between the variables measured are reported. The statistics of cluster size diversity has important consequences in the statistical description of the Universe as a complex system. [S1063-651X(99)08709-7]
\end{abstract}

PACS number(s): 05.40.-a, 05.10.Ln, 05.70.Fh, 05.70.Jk

\section{INTRODUCTION}

Diversity is probably one of the main features of interest in many natural phenomena. The richness of patterns and behaviors in many dynamical systems have drawn the interest of many researchers in various branches of science, e.g., in biology [1], evolution [2,3], self-organization and cellular automata $[4,5]$, fractals $[6,7]$, and nonequilibrium phenomena $[8,9]$. The concept of diversity is universal in various fields of human knowledge. Similarly, the notion of complexity is present in a variety of natural phenomena and can be associated with several properties of a system. In many situations, complexity is related to the diversity of size scales. Presently, there is no generally accepted definition of a complexity measure, even though several studies have been made in attempt towards a complexity measurement [1015]. However, these studies agree that there should exist a complexity measure, which attains its maximum value for configurations in between a completely ordered and disordered state.

In the last few years, diversity of size or mass has been studied in several dissipative processes and cellular automata that generate a distribution of fragments and are of interest in physics $[16,19]$, chemistry [8], biology [18], and ecology $[16,17]$. Recently, Gomes and co-workers [14,15] proposed the diversity of size of fragments as a measurement for complexity in aggregation and fragmentation processes. These studies are of interest since they capture the idea of a complex configuration in between a completely connected structure (initial state, ordered) and completely disconnected state with no structure (disordered).

In this paper, we are interested in the diversity and the scaling behavior of cluster sizes on randomly occupied lattices. Note the similarity with the problem of aggregation and fragmentation processes, even though in the system considered there are no dynamics involved. In fragmentation, the process starts with a single connected cluster that is broken up into various smaller clusters following a specific algorithm. This procedure ends when all of the mass is consumed or just small and uniform clusters are present, depending if the fragmentation dynamics follows a consumption of mass or a conservation of mass process [8]. On the other hand, aggregation is a process opposite to fragmentation, where starting from a empty lattice one generates an aggregate following certain occupation dynamics [9]. In both systems, the processes evolve through time differently from randomly occupied lattice in which the measurements are taken over the probability.

Another point of interest is that randomly occupied lattices are used as initial configuration in most of the studies on cellular automata. In this way, a proper understanding of the clustering at the initial configuration may give more insight into some aspects of their dynamics, as shown in $[16,17]$. Moreover, the system analyzed is closely related to the percolation problem [24], even though in this case we are interested in the behavior of the number and the diversity of clusters size other than the spanning or percolation cluster. Still some connections exist between the two models, and we show a relation between the point of geometric phase transition, i.e., the appearance of the spanning cluster, and the point of maximum diversity and maximum entropy.

Recently, we reported the occurrence of critical probabilities associated with the maximum of diversity, $D_{\max }$, and the maximum number of clusters, $N_{\max }$, on a randomly occupied square lattice $[20,21]$. In this paper, we extended those results and determined the values of the critical probabilities for lattices with higher dimensionality. We show the behavior of the cluster size diversity and total number of clusters on these lattices and the scaling relations between the variables of interest. Also an entropic measurement based on the probability that an arbitrary occupied site is part of an $s$-site cluster is derived.

The structure of this paper is as follows: in Sec. II we describe and mathematically define a quantitative measurement that characterizes complexity in this system. In Sec. III we describe the computer simulations. In sec. IV the relation between cluster size diversity and percolation, describing the tuning effect in diversity, the entropic measurement of the system, and the critical probability associated with the variables measured. In Sec. V we analyze the scaling relations and offer some evidences on the statistical description of the universe as complex systems. Finally, in Sec. VI we give concluding remarks. 


\section{DIVERSITY AND COMPLEXITY}

Diversity is one of the most remarkable and important characteristics in nature. It manifests in several ways and is characterized by a range of different kinds of patterns exhibited by a system. To define diversity one must work with a function of interest such as a physical property. This function defines the type of pattern structures analyzed in the system and is generally defined a priori by an observer. As a result, diversity as a measurement of complexity presents an inherent subjective constraint.

On randomly occupied lattices diversity can be defined as the amount of different cluster sizes at a fixed probability of occupation $p$. Here a cluster is defined as a collection of occupied sites connected by the nearest neighbors. The system in consideration has no dynamics and no correlation between the sites; consequently, the structural complexity can be well defined as the amount of different cluster sizes on the lattice. Moreover, other definitions could be adopted, such as a differentiation on cluster forms or lattice animals $[21,22]$.

The mathematical definition for the total number of clusters is given by

$$
N(p)=\left\langle\sum_{s} N(s, p)\right\rangle,
$$

and for the diversity of the mass of a cluster,

$$
D(p)=\left\langle\sum_{s} \Theta[N(s, p)]\right\rangle .
$$

In these expressions, $N(s, p)$ is the number of clusters of size $s$, in a single experiment. For occupation probability $p$; $\Theta(x)$, the Heaviside function is defined as $\Theta(x)=1$ if $x$ $>0$ and $\Theta(x)=0$ otherwise, and the average $\langle\ldots\rangle$ is over different experiments. This definition is suitable because it is a quantifiable macroscopic physical measurement.

Even though cluster size diversity is an appropriate measurement of complexity for the system studied, we should keep in mind that it has limitations as a general definition of complexity, since the pattern structure is defined a priori and no correlation between these patterns are taken into account.

\section{SIMULATIONS}

We report the behavior of the number of clusters and diversity of cluster size by performing Monte Carlo simulations on Euclidean lattices with dimensions 1 to 6 . The lattices were randomly occupied with probability ranging from $p=0.01$ to $p=0.99$ with steps of 0.01 . However, in the region near the maximum of cluster size diversity and the maximum number of clusters, we used a more precise value of $p$ with steps of 0.002 . For the one-dimensional case the probability was extended up to $p=0.99999$. In these simulations, we have generated lattices with total number of sites varying from the order of $10^{3}$ to $10^{7}$ with averages taken on 6000 to 50 experiments.

We applied the well-known Hoshen-Kopelman algorithm [23] to identify the different cluster sizes, using an IBM RISC computer. The present simulations do not require memory allocation for the whole lattice, since the generated lattice does not follow a dynamics (correlation in time), nor do the sites depend on another site state (correlation in space). Consequently, it was possible to perform simulations in very large lattice sizes without great computational demand.

\section{CLUSTER SIZE DIVERSITY AND PERCOLATION}

\section{A. Tuning effect}

From the point of view of clusters generated by randomly occupying the sites of a lattice, the probability of occupation $p$ determines the structures of the clusters in the system. Here, a low value of $p$ will generate a lattice occupied with various small disconnected clusters of equal size as shown in Fig. 1(a), while with $p$ close to 1 a totally connected single cluster appears, Fig. 1(d). An intermediate value of $p$ gives a configuration with maximum cluster size diversity, Fig. 1(b). The size of the lattice $L$ determines the possible amount of cluster diversity, since increasing the lattice size results in an increase in the range of the cluster size scales. Consequently, by changing the parameters $L$ and $p$ we attain a configuration not only with great diversity, but also a state in which a spanning cluster percolates the lattice.

In Fig. 2 we show the log-log plot of the diversity density $D / L^{d}$ versus probability of occupation for dimensions 1 to 6 and different values of $L$, in which $d$ is equal to the dimension of the lattice. Diversity increases exponentially, attains a maximum, and then decreases. For every curve the maximum diversity was obtained in a defined probability. The shape of these curves shows that the rate of increase in diversity is higher at the maximum region. An increase on the size $L$ of the system implies a smaller region of $p$ associated with a high diversity or complexity. Consequently, cluster size diversity can be tuned by parameters $L$ and $p$. Also, an increase in the dimensionality of the lattices implies an increase of the connectivity of the system. For this reason, as the dimensionality increases, the value of $p$ where the diversity is maximum, decreases.

In these plots, we also observe a phase where the diversity density curves attain a constant value. This phase is characterized by a large connected cluster with some very small clusters, Fig. 1(c). Then the diversity of clusters decreases until it reaches the unity value, as shown in Fig. 1(d), which causes the flat regions in the diversity density plots, as shown in Fig. 2. These flat regions are due to a finite-size effect. In these plots we can observe that as the dimension of the system increases the flat regions become more evident. However, as the size of the lattice gets larger these regions become smaller. Consequently, with the increase of the system size, those regions should disappear.

The total number of clusters $N$ was also measured in these simulations. Figure 3 shows the $\log$ - $\log$ plots of $N$ normalized by $L^{d}$ so that all curves collapse irrespectively of the lattice size, except for $p$ close to one. This is also due to finite-size effects, since as the lattice size $L$ increases those regions tends to vanish. Similar to the diversity density plots, the flat regions should disappear with the increase of the lattice size. Also, we can observe that the ratio $N / L^{d}$ increases with $p$, reaches a maximum, and decreases afterwards. However, the behavior of these curves are distinctively different from the diversity density plots, which shows a peaked region around the maximum. 
(a)

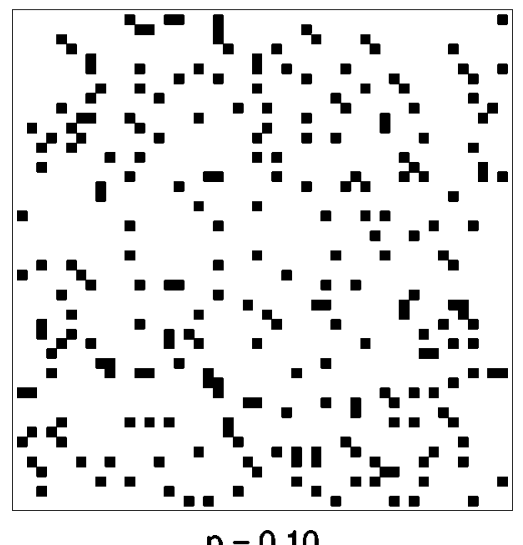

(c)

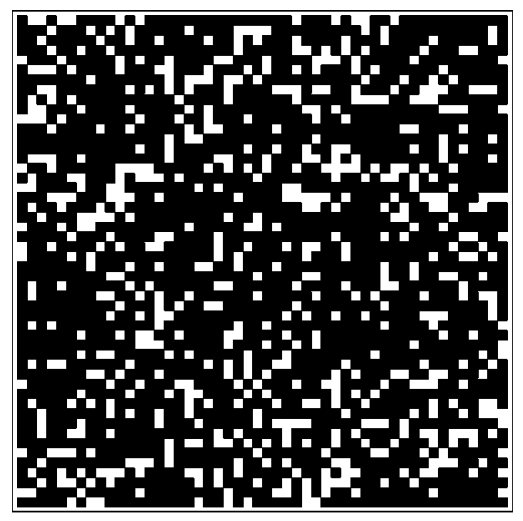

$p=0.75$ (b)

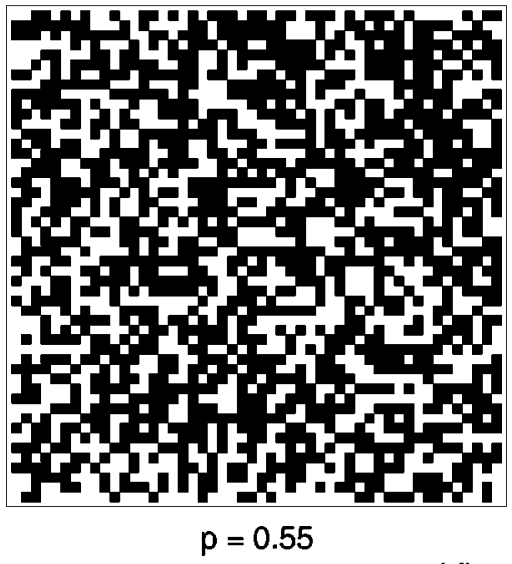

(d)

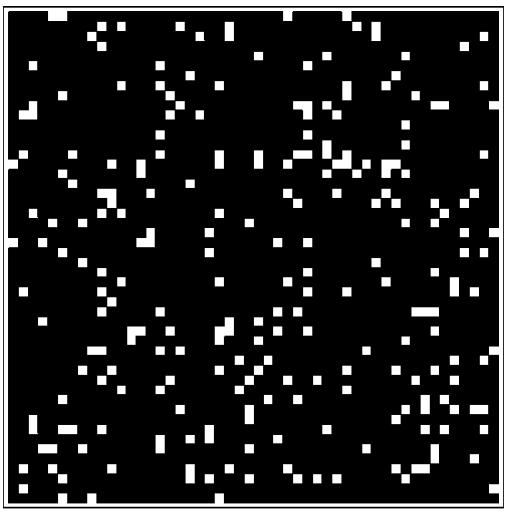

$p=0.90$
FIG. 1. Snapshots of a 50 $\times 50$ square lattice randomly occupied with probabilities $p=0.10$, $0.55,0.75$, and 0.95. (a) For $p$ $=0.10$ the lattice is occupied with various small and disconnected clusters of the same size. (b) At $p=0.55$ a configuration with maximum cluster size diversity emerges. (c) For $p=0.75$ the lattice is characterized by a main connected cluster with some very small clusters. (d) For $p=0.90$ a single totally connected cluster appears.

\section{B. Entropy}

The entropy of the system can be defined as a function of the probability that an occupied site is part of an $s$-site cluster. In this system, the number of $s$-cluster per lattice site or normalized cluster number $n_{s}$ is defined as

$$
n_{s}=\frac{\text { number of cluster of size } s}{\text { total number of lattice sites }} .
$$

So, the probability that an arbitrary occupied site belongs to a cluster containing $s$ sites is given by

$$
w_{s}=\frac{s n_{s}}{\sum_{s} s n_{s}} .
$$

Consequently, the entropy of the system is defined as

$$
H=-\sum_{s} w_{s} \ln w_{s} .
$$

Figures 4 and 5 show the entropy versus the probability of occupation for the one and two-dimensional lattices, respectively. The value of $H$ increases exponentialy until the percolation threshold; at this point, a geometric phase transition occurs and the spanning cluster dominates the system. To find the probability associated with the maximum entropy, we used $p$ with steps of 0.0001 and 0.002 near $P\left(H_{\max }\right)$ for $d=1$ and $d=2$, respectively. The inset of these figures shows the plots of $P\left(H_{\max }\right)$ versus $1 / L$. In this way, we determine the probability of maximum entropy at the thermodynamic limit, which is given by $P_{c}\left(H_{\max }\right)=0.997 \pm 0.005$, for the one-dimensional case and $P_{c}\left(H_{\max }\right)=0.57 \pm 0.04$, for the square lattice.

In the simulations of the one-dimensional lattice the maximum entropy and maximum diversity of cluster size at the thermodynamic limit is obtained at $p$ approximately equal to one. In an ideal situation, the probability of the maximum diversity for the one-dimensional case can be calculated exactly. Suppose a one-dimensional lattice with the maximum diversity of cluster size. Such a lattice would have clusters with sizes $1,2,3,4,5, \ldots$ so that the total number of occupied sites is given by

$$
1+2+3+4+5+\cdots=\frac{D(D+1)}{2} .
$$

The number of empty sites is given by $(D-1)$, since one site is required to separate each cluster. $D$ in these equations is equivalent to the diversity of the lattice. This leads to

$$
\frac{D(D+1)}{2}+(D-1)=L,
$$

since the total number of occupied sites plus the total number of empty sites yield the size of the lattice. The probability to find an occupied and an empty site in the lattice is given, respectively, by 

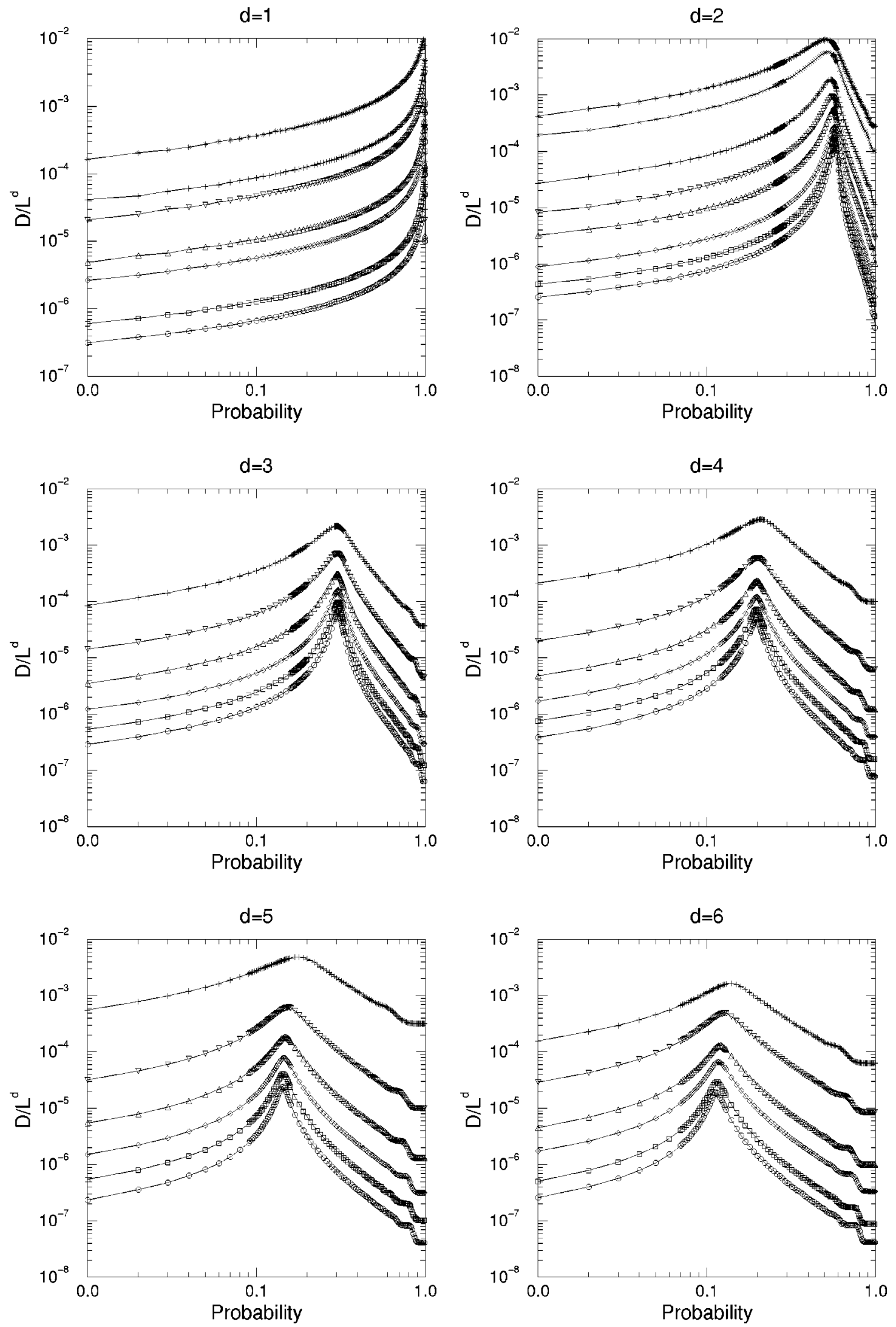

FIG. 2. Log-log plot of the cluster size diversity density, $D / L^{d}$, as a function of the probability of occupation for dimensions 1 to 6 . The following values of $L$ were used in these simulations. For $d=1 ; L=1 \times 10^{7}(\bigcirc), 5 \times 10^{6}(\square), 1 \times 10^{6}(\diamond), 5 \times 10^{5}(\triangle), 1 \times 10^{5}(\nabla), 5$ $\times 10^{4}(+)$, and $1 \times 10^{4}(*)$. For $d=2 ; L=4000(\bigcirc), 3000(\square), 2000(\diamond), 1000(\triangle), 600(\nabla), 300(+), 100(\times)$, and $60(*)$. For $d=3 ; L$ $=250(\bigcirc), 200(\square), 150(\diamond), 100(\triangle), 60(\nabla)$, and 30(+). For $d=4 ; L=60(\bigcirc), 50(\square), 40(\diamond), 30(\triangle)$, 20( $\nabla)$, and 10(+). For $d$ $=5 ; L=30(\bigcirc), 25(\square), 20(\diamond), 15(\triangle), 10(\nabla)$, and 5(+). For $d=6 ; L=17(\bigcirc), 15(\square), 12(\diamond), 10(\triangle), 7(\nabla)$, and 5(+). 

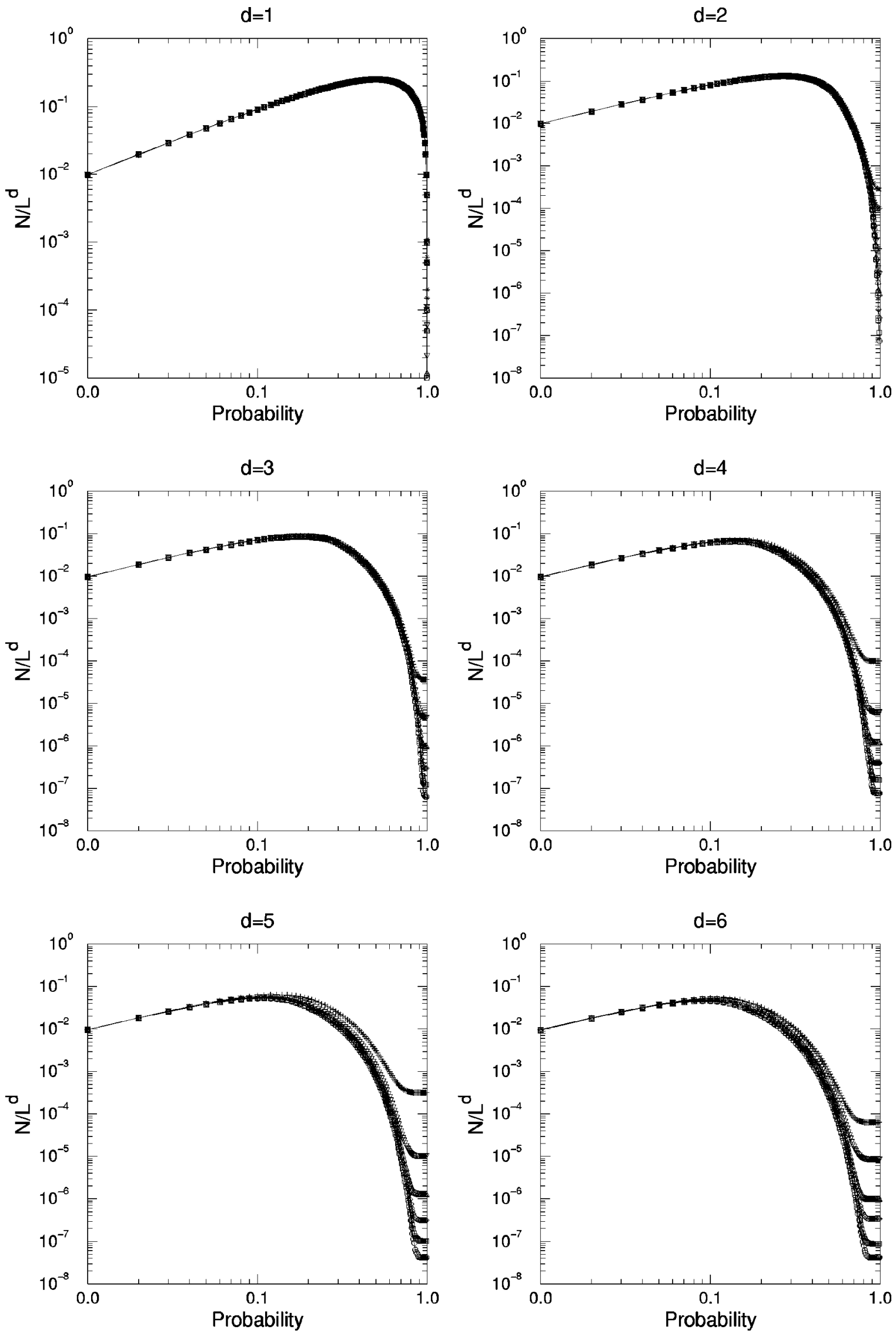

FIG. 3. The log-log plot of the collapsing curves $N / L^{d}$ as a function of the probability of occupation for different dimensions and lattice sizes. A flat region is observed for a value of $p$ close to 1 . These flat regions became larger with an increase in the dimensionality of the system. However, for a larger lattice size these regions became smaller, indicating that this is due to finite-size effects, therefore, those regions should disappear as the size of the lattice becomes larger. 


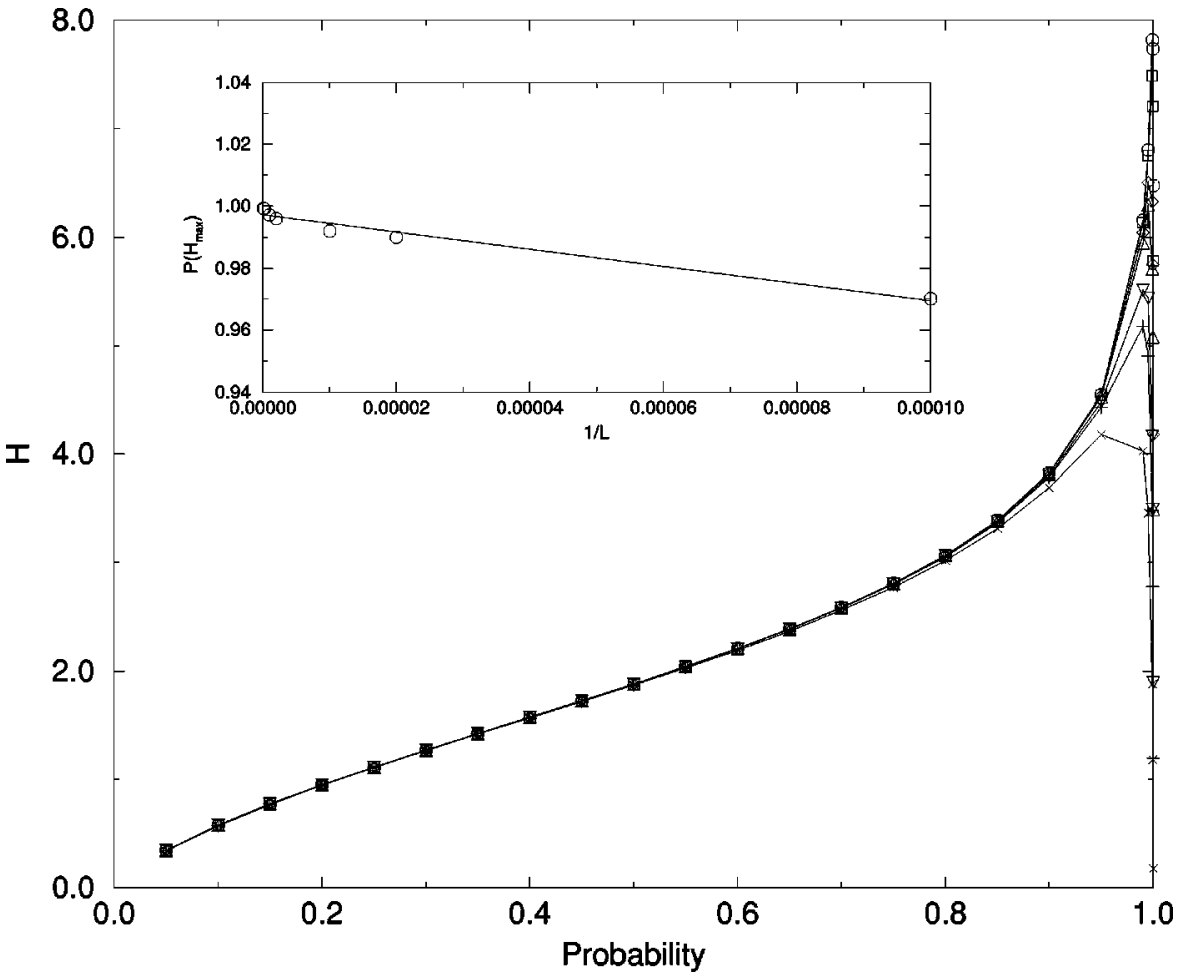

FIG. 4. Plot of the entropy versus the occupation probability for the one-dimensional lattice. The inset shows the plot of the probability of the maximum entropy as a function of $1 / L$. The following values of $L$ were used in these simulations: $\quad L=1 \times 10^{7}(\bigcirc), \quad 5$ $\times 10^{6}(\square), \quad 1 \times 10^{6}(\diamond), \quad 5$ $\times 10^{5}(\triangle), \quad 1 \times 10^{5}(\nabla), \quad 5 \times 10^{4}$ $(+)$, and $1 \times 10^{4}(*)$, with averages taken on 1000 to 50 experiments.

$$
p=\frac{D(D+1)}{2 L}
$$

$p=\frac{D+1}{D+3-\frac{2}{D}}$

and

$$
1-p=\frac{D-1}{L}
$$

so that,

Substituting the equations and taking the limit we have

$$
\lim _{D \rightarrow \infty} p=1
$$

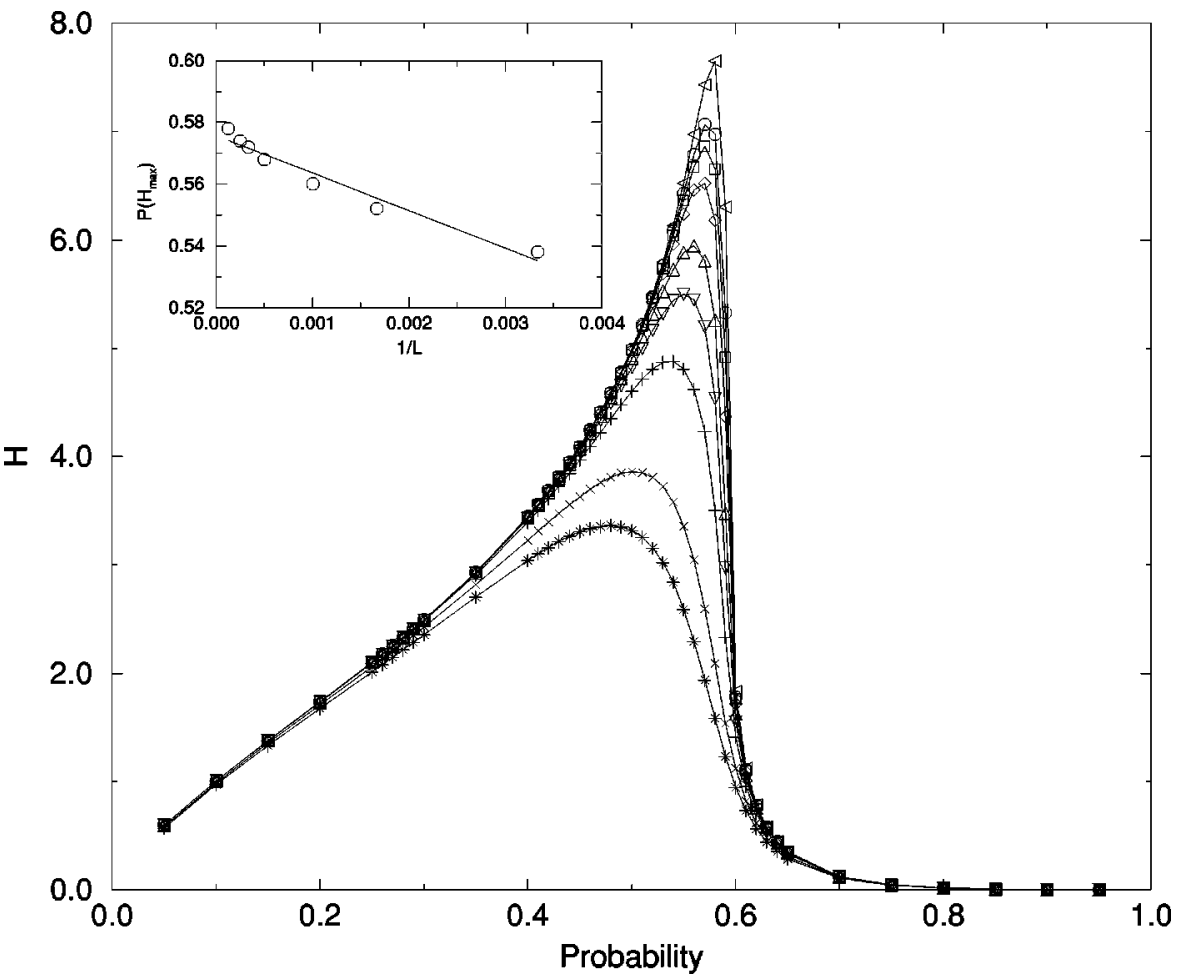

FIG. 5. Similar to Fig. 4 but for the two-dimensional lattice. The following values of $L$ were used in these simulations: $L$ $=8000(\triangleleft), 4000(\bigcirc), 3000(\square)$, $2000(\diamond), \quad 1000(\triangle), \quad 600(\nabla)$, $300(+), \quad 100(\times)$, and $60(*)$, with averages taken on 6000 to 20 experiments. Note that the curves show a sharp transition after $p_{c}$, since most of the occupied sites belong to the percolation clusters. 

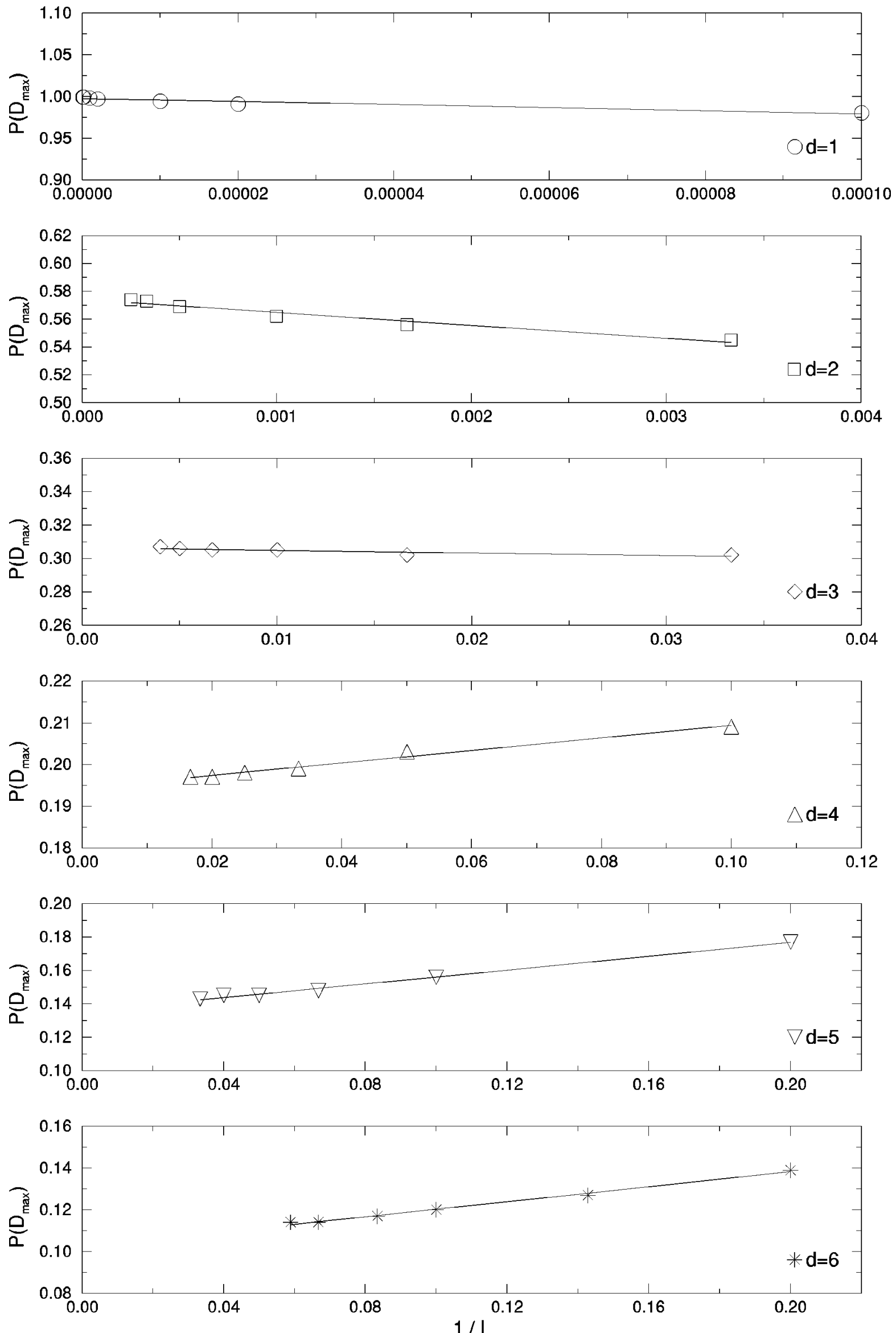

FIG. 6. Plot of the linear fit for $p\left(D_{\max }\right)$ as a function of $1 / L$ for the various dimensions. The intercept of the linear fit gives the value for critical probability at the thermodynamic limit. 

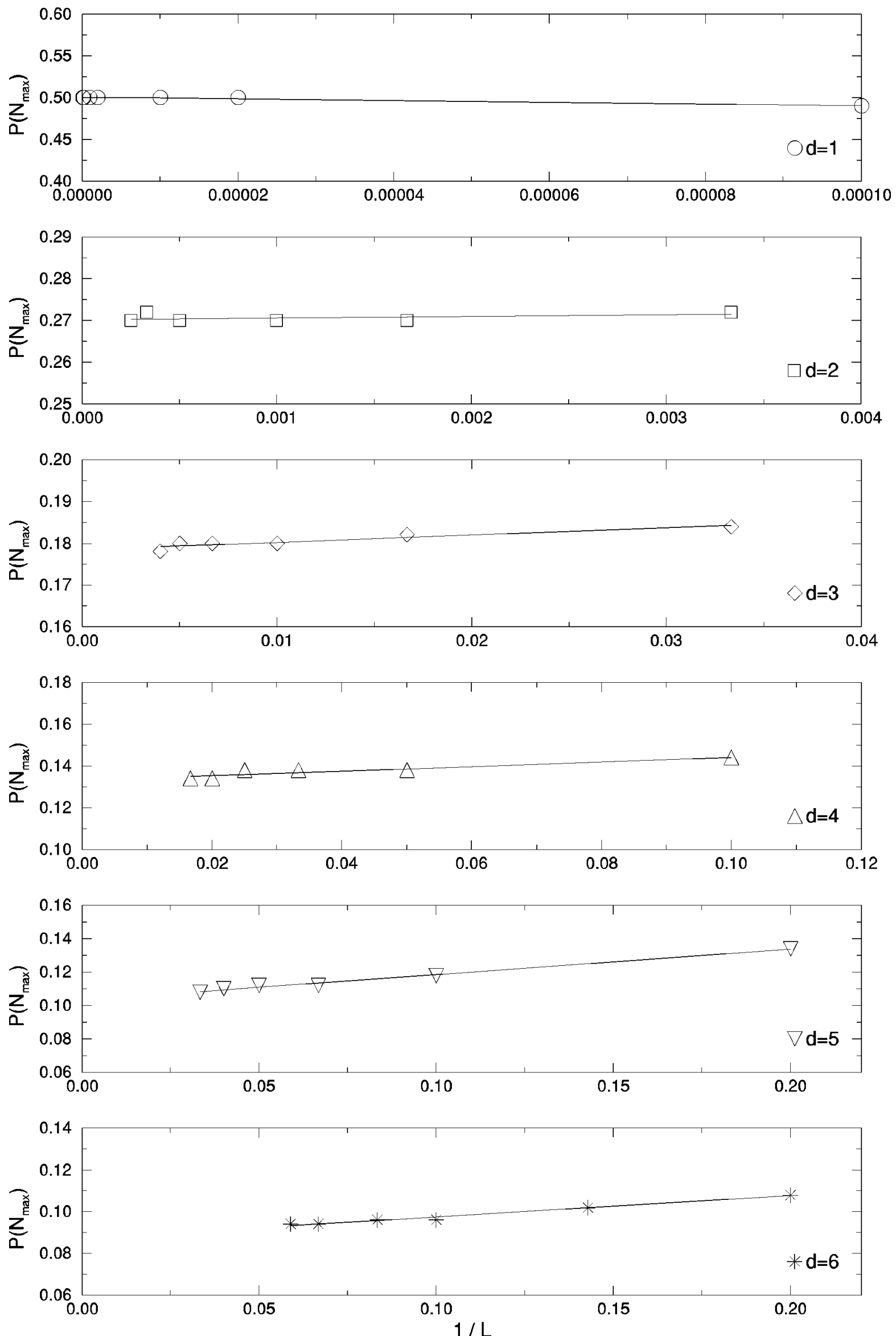

FIG. 7. The linear fit for $p\left(N_{\max }\right)$ as a function of $1 / L$ for the various dimensions. These plots are similar as in Fig. 4, but for the maximum number of clusters. 
TABLE I. The values of the critical probabilities for the maximum number of cluster size $p_{c}\left(N_{\max }\right)$, the maximum cluster size diversity $p_{c}\left(D_{\max }\right)$, the percolation threshold $p_{c}$, and the difference between the probability of maximum diversity and the percolation probability $\Delta p$, for the different dimensions investigated. $\Delta p$ shows that $p_{c}\left(D_{\max }\right)$ and $p_{c}$ are statistically the same.

\begin{tabular}{ccccc}
\hline \hline$d$ & $p_{c}\left(N_{\max }\right)$ & $p_{c}\left(D_{\max }\right)$ & $p_{c}{ }^{\mathrm{a}}$ & $\Delta p$ \\
\hline 1 & $0.501 \pm 0.002$ & $0.998 \pm 0.005$ & 1 & $0.002 \pm 0.005$ \\
2 & $0.270 \pm 0.002$ & $0.574 \pm 0.005$ & 0.592746 & $0.019 \pm 0.005$ \\
3 & $0.178 \pm 0.002$ & $0.307 \pm 0.007$ & 0.3116 & $0.005 \pm 0.007$ \\
4 & $0.133 \pm 0.002$ & $0.194 \pm 0.007$ & 0.197 & $0.003 \pm 0.007$ \\
5 & $0.103 \pm 0.002$ & $0.136 \pm 0.007$ & 0.141 & $0.005 \pm 0.007$ \\
6 & $0.087 \pm 0.002$ & $0.102 \pm 0.009$ & 0.107 & $0.005 \pm 0.009$ \\
\hline
\end{tabular}

${ }^{a}$ Values obtained from Ref. [24].

(a)

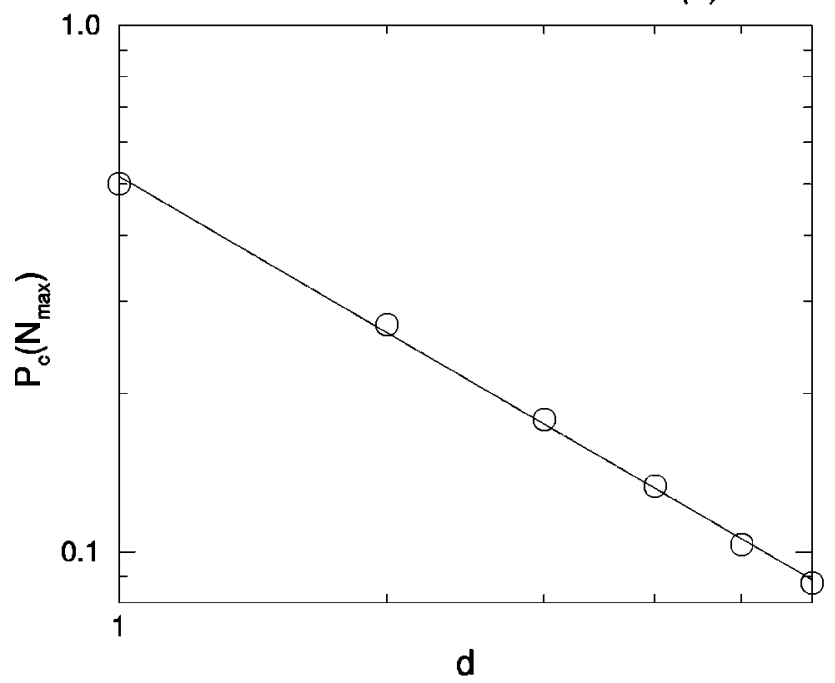

(c)

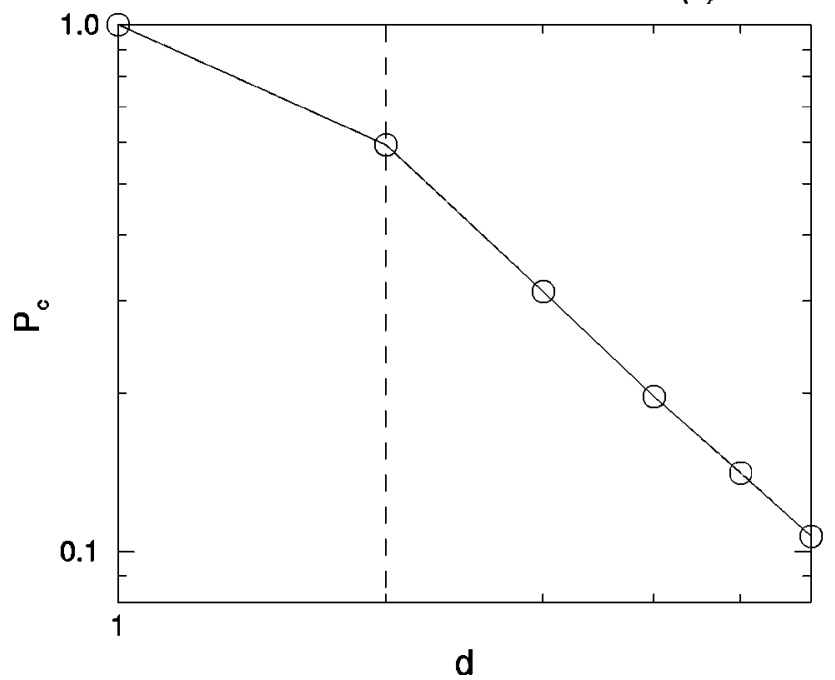

Consequently, if we consider a one-dimensional lattice with $D \rightarrow \infty$, which implies that $L$ also goes to infinity, the maximum diversity is obtained at the exact value of $p=1$. That is the same value for the site percolation threshold for the one-dimensional case [24]. The same kind of mathematical reasoning could be applied to the other dimensions. The total number of occupied sites could be estimated in the same way as in the one-dimensional case. However, the empty sites will depend on the external perimeter of the clusters, which will lead us to a similar problem as of calculating the general exact solution for the percolation problem in $d$-dimension, see [24]. Furthermore, we leave for future study an exact solution for the Bethe lattice, since for the percolation problem an exact solution exists and this lattice corresponds in some sense to infinite dimensionality.

(b)

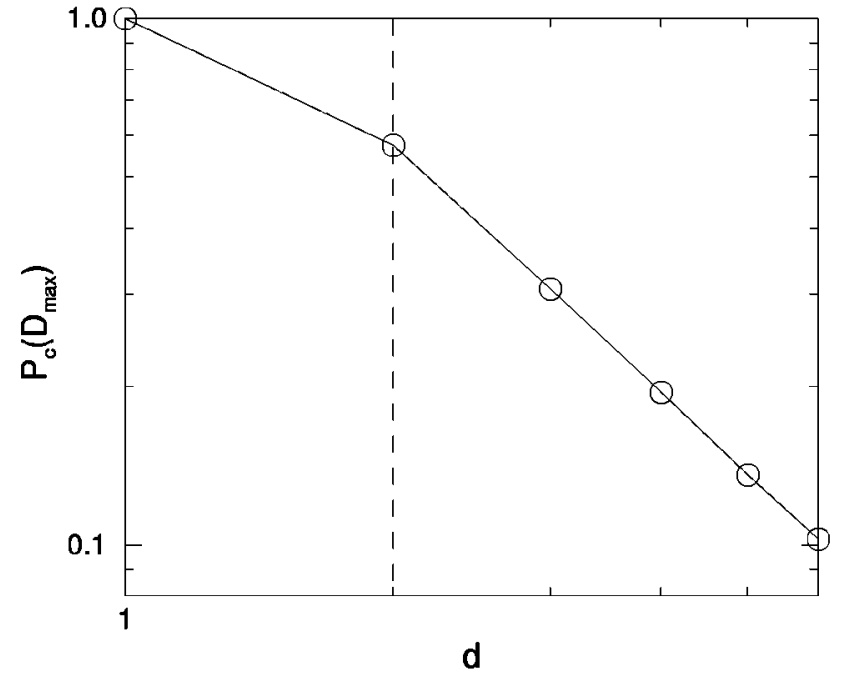

(d)

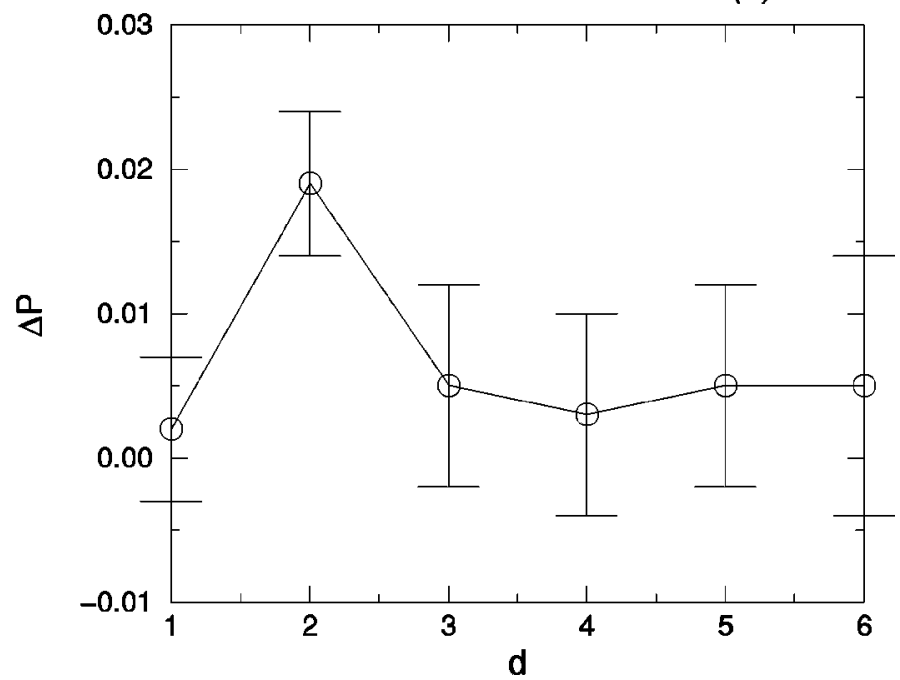

FIG. 8. (a) Plot of the critical probability for maximum number of cluster, $p_{c}\left(N_{\max }\right)$, versus the lattice dimensionality. (b) $p_{c}\left(D_{\max }\right)$ versus $d$, the fit shown in Eq. (11) was taken for $d \geqslant 2$. (c) The plot of the critical probability for the percolation cluster as a function $d$, similar to (b). (d) Plot of the difference between $p_{c}\left(D_{\max }\right)$ and $p_{c}$, defined as $\Delta p$ versus $d$. 


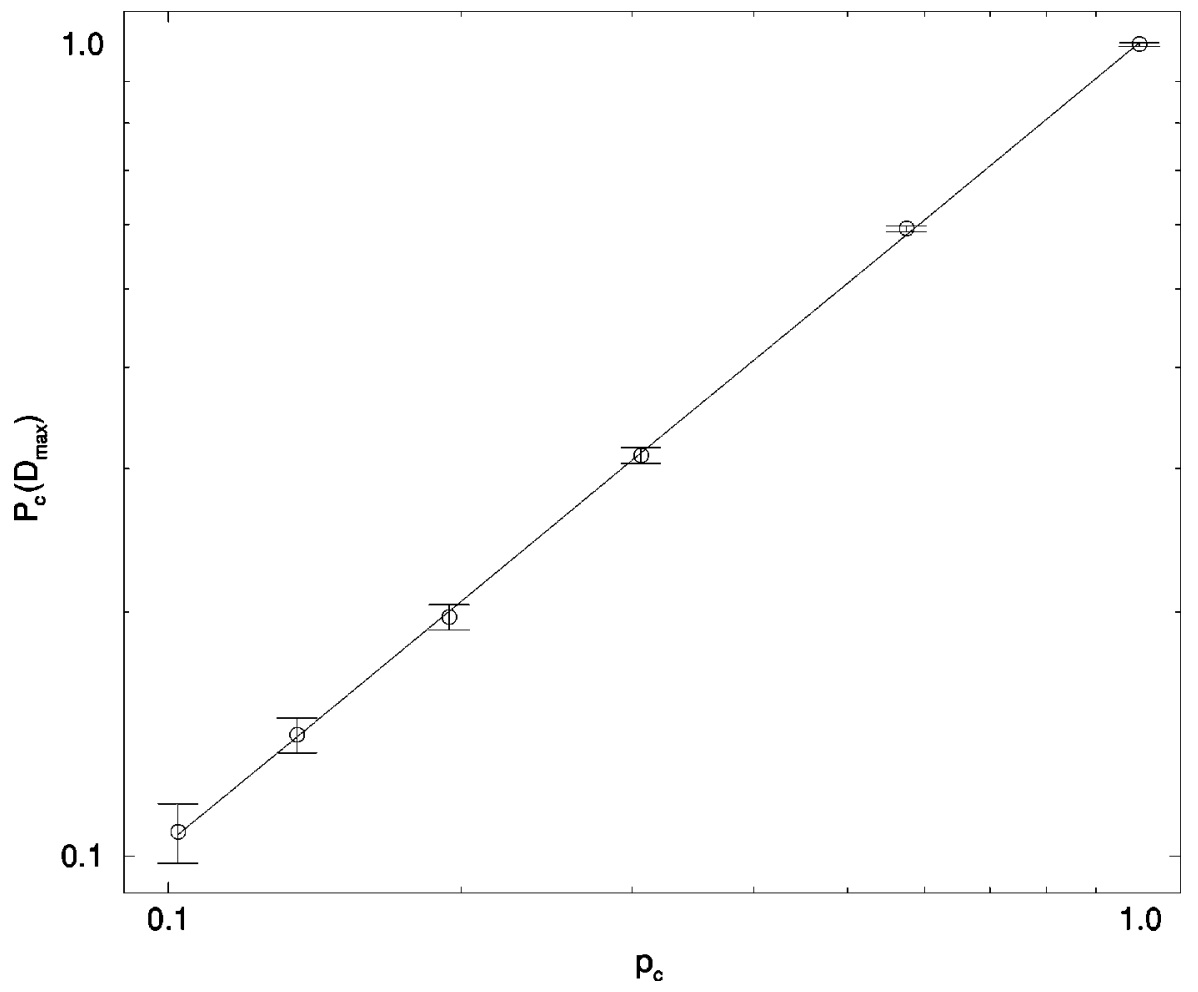

FIG. 9. The scaling relation of $P_{c}\left(D_{\max }\right)$ versus $p_{c}$, which indicates that these two critical probabilities have the same value.

\section{Critical probabilities}

Finite-size scaling analysis was used to determine the critical probabilities associated with $D_{\max }$ and $N_{\max }$ at the thermodynamic limit. In Fig. 6 we show the plots of $p\left(D_{\max }\right)$ versus $1 / L$. Using a linear fit of the form

$$
p\left(D_{\max }\right)=\Omega L^{-1}+\Pi,
$$

we obtained $\Pi$, the critical probabilities at $L \rightarrow \infty$ and $\Omega$, which is the slope of the curves. The constant $\Omega$ determines the influence of the size of the system on the probability of maximum diversity. Its importance is discussed in the next section. The same analysis was used for the maximum number of cluster size as shown in Fig. 7. Table I shows the values of $p_{c}\left(D_{\max }\right)$ and $p_{c}\left(N_{\max }\right)$ at the thermodynamic limit, the percolation threshold $p_{c}$, and the difference between $p_{c}\left(D_{\max }\right)$ and the percolation threshold $\Delta p$, for the various dimensions. The values of $\Omega$ are shown in Table II. The values obtained for $\Delta p$ are always positive since after the percolation threshold less space is left for the appearance of different clusters; consequently, diversity attains a lower value. On a finite lattice, the maximum of cluster size diversity appears before the $p_{c}$. This can clearly be seen in the one-dimensional case.

In Fig. 8(a) we show the plot of $p_{c}\left(N_{\max }\right)$ versus the dimension of the system, which yields the following fit:

$$
p_{c}\left(N_{\max }\right)=0.516 d^{-0.986 \pm 0.003} \text {. }
$$

Figures $8(\mathrm{~b})$ and $8(\mathrm{c})$ show the plots of $p_{c}\left(D_{\max }\right)$ and the critical probability for the percolation cluster $p_{c}$ that, respectively, follows the fits

$$
p_{c}\left(D_{\max }\right)=1.719 d^{-1.575 \pm 0.005}
$$

and

$$
p_{c}=1.736 d^{-1.560} \text {. }
$$

The critical probability for the one-dimensional case is not included in these fits, since the curves clearly show a different behavior for $d$ greater than 2. These two fits are very similar, although the values of $p_{c}\left(D_{\max }\right)$ obtained are smaller than the percolation threshold. The values of $p_{c}$ were obtained from Ref. [24]. In Fig. 8(d) we have the plot of the difference of these probabilities for various dimensions, all the points are very close to zero. The error bars show that statistically the two points are the same. Figure 9 shows the plot of the scaling relation between the two critical probabilities, giving

$$
p_{c}\left(D_{\max }\right)=0.992 p_{c}^{1.013 \pm 0.005}
$$

There is an intrinsic difficulty in finding an exact solution for the critical probabilities for the percolation problem. This also seems to be the same case for the probability of maximum diversity.

The one-dimensional percolation problem represents a one-side phase transition with critical probability equal to 1 . Similarly, the probability of maximum diversity appears to be at a specific point. For the one-dimensional case, both critical probabilities were obtained at the same point, as shown in the last section. For higher dimensions they are shown to be statistically equivalent. Therefore, on the light of these evidences, it is reasonable to conjecture that the probability of maximum cluster size diversity and of the percolation threshold are the same. 


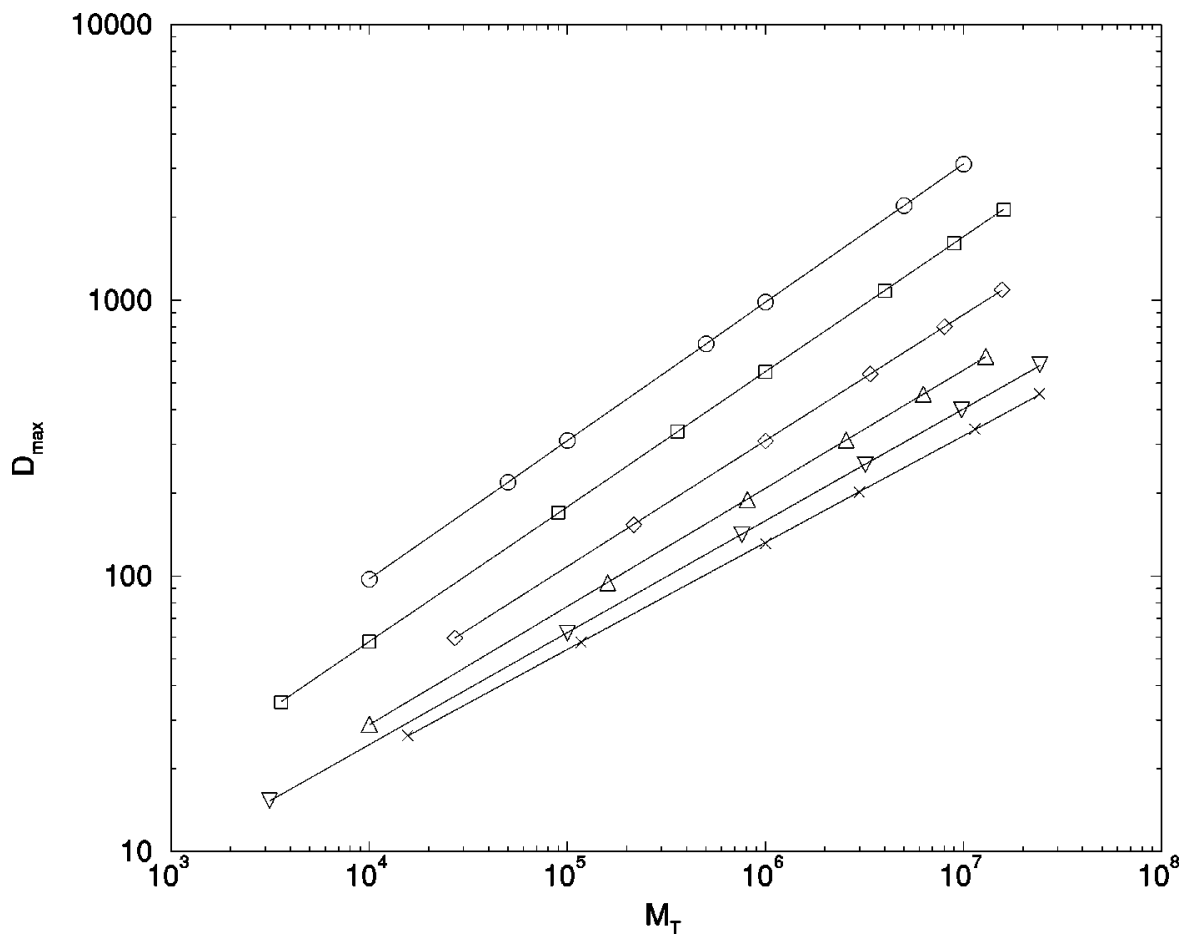

FIG. 10. Log-log plot of $D_{\max }$ versus $M_{T}$ leading to the scaling $D_{\max } \sim M_{T}^{\theta}$ for $d=1(\bigcirc), 2(\square)$, $3(\diamond), 4(\triangle), 5(\nabla)$, and $6(\times)$. The values of the exponent $\theta$ are listed in Table II.

\section{SCALING AND COMPLEX SYSTEMS}

\section{A. Scaling relations}

In Fig. 10, we plotted $D_{\max }$ versus $M_{T}$ for different lattice sizes and dimensions. Here $M_{T}$ is the total mass capacity of the system or volume, which can also be defined as $L^{d}$. The maximum diversity follows the scale

$$
D_{\max } \sim M_{T}^{\theta},
$$

the values of $\theta$ for each dimension are shown in Table II.

By fixing a probability $p$ and taking the scaling relation of $D$ versus $L$ we obtain the exponents $\beta$ as a function of $p$,

$$
D(L, p) \sim L^{\beta(p)}
$$

In Fig. 11, we show the plot of this value. $\beta$ increases with $p$, reaches a maximum, and decreases afterwards, similarly to the diversity plot. This indicates that the rate of increase in diversity for different values of $L$ is higher in the maximum region. It is interesting to note that for $d \geqslant 3$, the value of $\beta$ does not fall constantly. For $p$ near the value of 1 ,

TABLE II. The values of $\Omega$ obtained from Eq. (9), the exponent $\theta$ from the scaling $D_{\max }$ versus $M_{T}$, and $\bar{\rho}$, the average values of $\rho$ from Eq. (19) for the different lattice dimensions.

\begin{tabular}{cccc}
\hline \hline$d$ & $\Omega$ & $\theta$ & $\bar{\rho}$ \\
\hline 1 & $-183 \pm 41$ & $0.50 \pm 0.01$ & $0.92 \pm 0.03$ \\
2 & $-9.32 \pm 0.07$ & $0.49 \pm 0.01$ & $0.40 \pm 0.03$ \\
3 & $-0.16 \pm 0.04$ & $0.46 \pm 0.02$ & $0.28 \pm 0.04$ \\
4 & $0.14 \pm 0.01$ & $0.43 \pm 0.02$ & $0.25 \pm 0.04$ \\
5 & $0.20 \pm 0.01$ & $0.41 \pm 0.03$ & $0.26 \pm 0.05$ \\
6 & $0.18 \pm 0.01$ & $0.39 \pm 0.04$ & $0.29 \pm 0.06$ \\
\hline \hline
\end{tabular}

we find a region in which the value of $\beta$ increases again creating a local maximum. This local maximum appears because of finite-size effects as a consequence of the flat regions shown in Fig. 2.

The fact that, for a fixed probability, $D(L)$ scale with $L$ to the exponents $\beta$ implies that the densities $D / L^{d}$ decay as $L^{(\beta-d)}$. Therefore, the diversity density varies not only according to the dimension of the system but also according to the size of the system. In this way, the diversity of clusters size measured on a finite system must be rescaled to correctly correspond to a large system. Figure 12 shows $D$ normalized by $L^{\beta}$ for all values of $p$, so that all the curves collapse irrespectively of size. Note that the curves are mainly characterized by two maxima for $d \geqslant 2$ and there is a change of behavior in these maxima in between dimensions 3 and 4.

\section{B. Sustainability of complex systems}

The structural complexity of the system in consideration has been characterized by the diversity of cluster sizes. Even though this definition has its limitations and is not a final measurement of complexity, it is a useful concept for the understanding of complex systems. Recent studies $[14,15]$ show that the statistics of cluster size diversity describes well the complexity in aggregation and fragmentation processes, and has important consequences for a statistical description of the universe. We extend these studies to the randomly occupied lattice, contributing to a better understanding of complex systems.

The relation between the maximum diversity and the total mass, $M_{T}$, or volume of the system was found to be $D_{\max }$ $\sim M_{T}^{\theta}$. Consequently, the production of diversity in different dimensions can be measured by the following ratio [14]:

$$
\rho=D_{\max }^{1 / \theta} / M_{T} .
$$



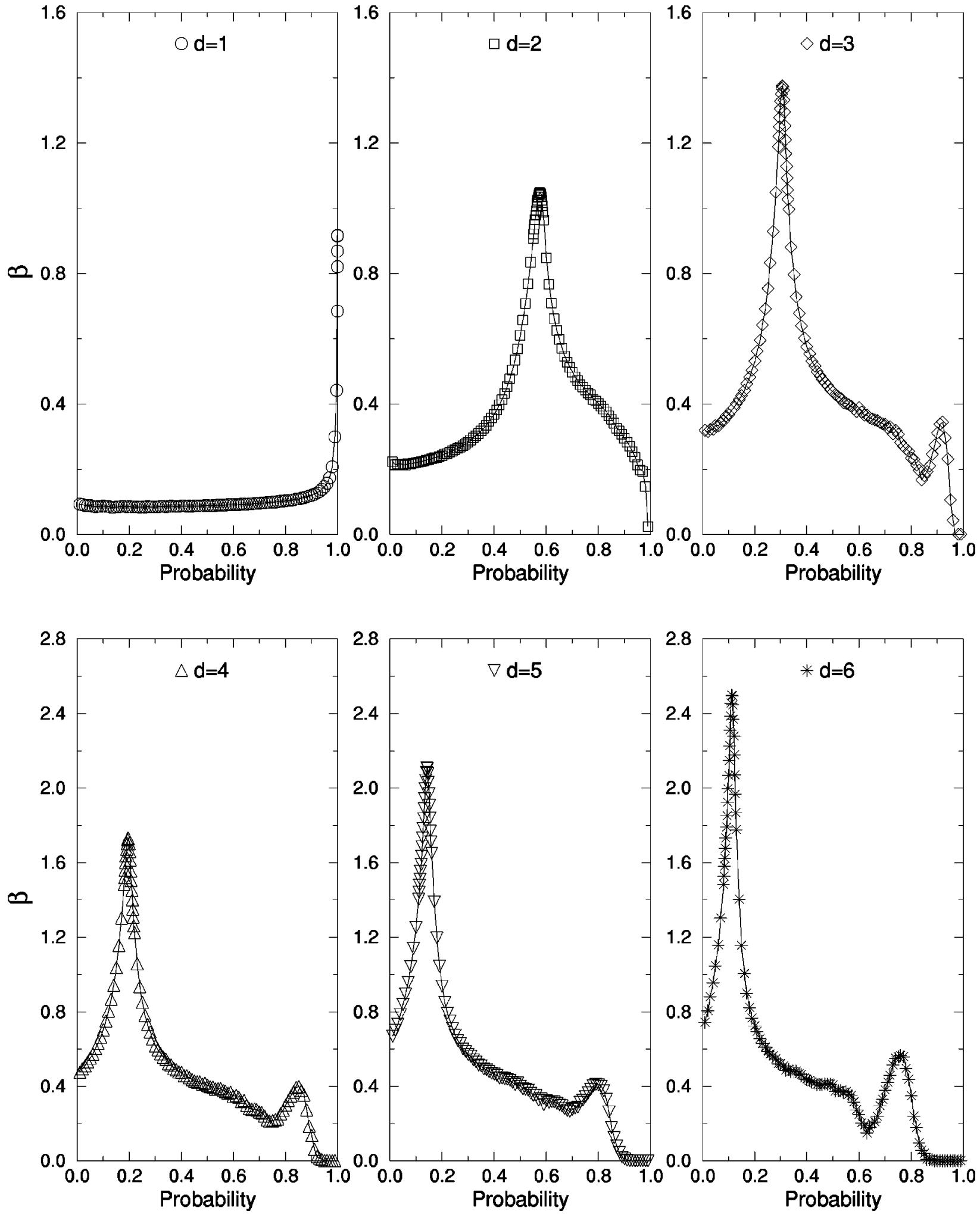

FIG. 11. The exponents $\beta$ as a function of $p$ for the various dimensions. The values of $\beta$ were obtained from the scaling relation $D(L, p) \sim L^{\beta(p)}$. Similarly to the diversity plots, $\beta$ increases with the probability attaining a maximum and decreases afterwards, except for $d=1$. Also, for $d \geqslant 3$ the curves show a local maximum, which is due to a finite-size effect associated with the flat region as shown in Fig 2.

In the present system, the diversity of cluster size depends only on the probability of occupation. Hence, $D_{\max }^{1 / \theta} / M_{T}$ measures the intrinsic capacity of the system to support diversity. The plot in Fig. 13(a) shows $\rho$ as a function of $d$, each point representing a different lattice size for different dimensions. In Table II, we listed the average values of $\rho$ for each dimension defined as $\bar{\rho}$.

Figure 13(a) shows that high space dimensions are less 

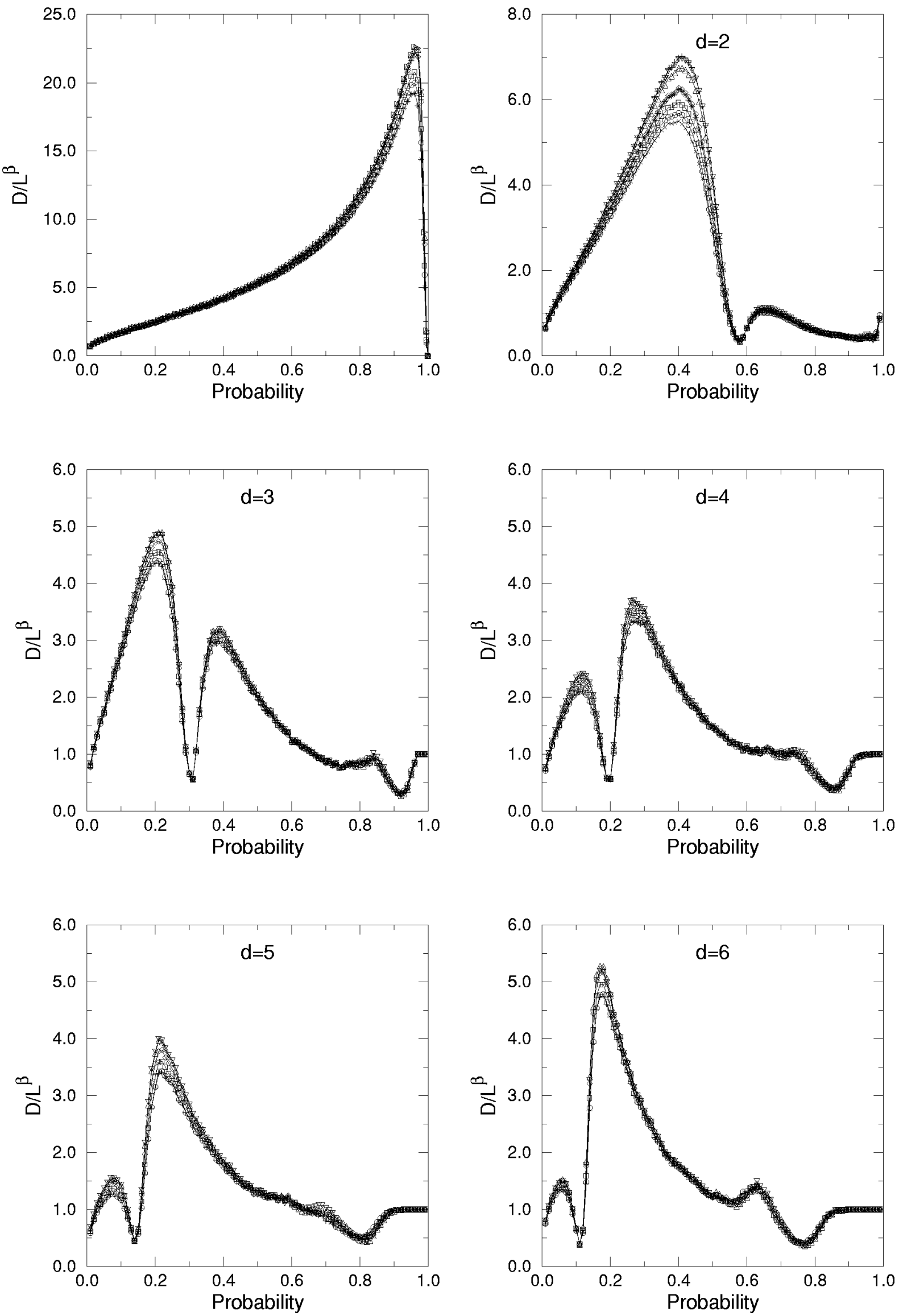

FIG. 12. The collapsing curves of the cluster size diversity normalized by $L^{\beta}$ as a function of $p$, for several lattice sizes and different dimensions. 

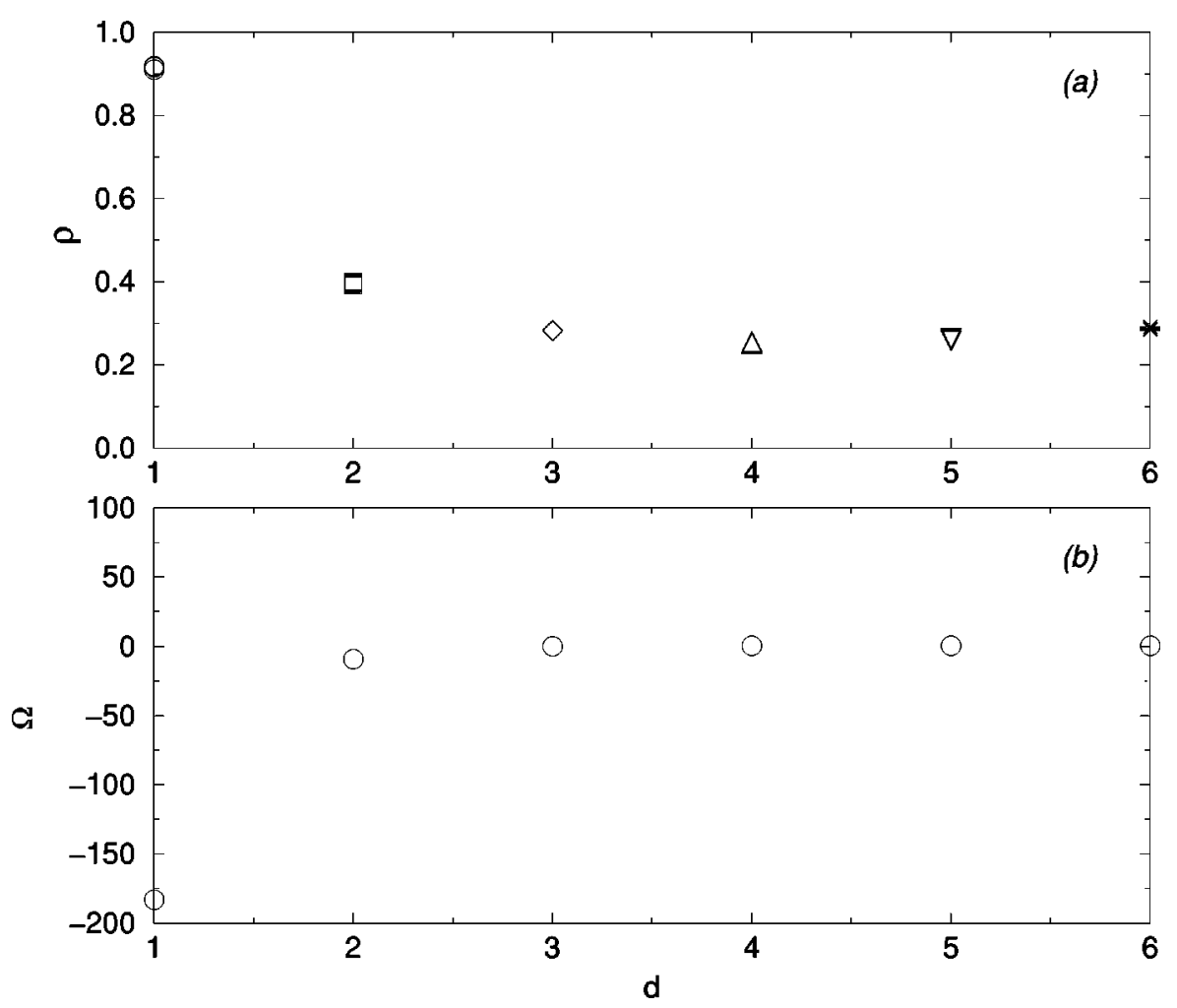

FIG. 13. (a) The plot of $\rho$ as a function of the lattice dimension. (b) Plot of $\Omega$ as a function of $d$. The sizes of the system used in these plots are listed in the caption of Fig 2. propitious to sustain diversity. A higher dimension means a higher connectivity, which implies an easier clustering of the objects, consequently, a decrease in the diversity of cluster size. As a result, lower dimensions favor the system to support a higher diversity or complexity. For $d \geqslant 3$ the values of $\bar{\rho}$ seem to attain a constant, possibly indicating that the capacity of the system to sustain diversity became invariant irrespective of the space dimension.

In Sec. IV we showed that the probability of maximum cluster size diversity and the percolation threshold, i.e., the point of geometric phase transition, are statistically the same. Figure 8(b) and Eq. (14) show that as the space dimension increases the probability to attain a higher diversity decreases. A lower probability of occupation implies less mass and, consequently, less energy. In this way, the higher the space dimension the less energy is necessary to attain a higher complexity.

In Eq. (12), $\Omega$ defines the relation between the probability of maximum diversity and the size, $L$, of the system. For the $1 d$ and $2 d$ case $\Omega$ is negative, while for the other dimensions it is close to zero; see Table II and Fig. 13(b). A negative $\Omega$ means that as $L$ increases the probability of maximum diversity increases, whereas $\Omega$ equal to zero means that the probability of maximum diversity does not depend on the system size. As mentioned before, a higher probability of occupation implies more mass and, consequently, more energy. As a result, for the dimensions 1 and 2, as the system size increases, the energy necessary to attain a more complex state increases. On the other hand, for $d \geqslant 3$ the energy necessary to create more complex structures is independent of the system size.

The sustainability of complex structures in the present system lies in the balance between the capacity of the system to support diversity and the energy necessary to attain a higher complexity. In agreement with $[14,15]$, our paper also indicates that $d=3$ seems to be optimal for the sustainability of a complex system. Our Universe is diverse and encompasses systems with enormous difference in length scale, from elementary particles to galaxies. We have shown that for $d \geqslant 3$ the energy to attain a higher diversity is independent of the system size. Also, a balance between lower dimensions that favors higher diversity, and higher dimensions that require less energy to attain high complexity, seems to be achieved at $d=3$. Consequently, a three-dimensional universe is ideal for the sustainability of complex systems.

\section{CONCLUSIONS}

In this paper, we described cluster size diversity as a measurement of complexity, showing that this measurement is suitable for systems that generate a statistical distribution of clusters. Nevertheless, diversity of patterns as a measurement of complexity has certain limitations, since it does not cover the organization or hierarchical structure of the system, which is an important component if one wants to have a full understanding of complex systems [12,25].

An interesting tuning effect in complexity for the randomly occupied lattices was observed. Also critical probabilities associated with the maximum of cluster size diversity and the maximum of number of cluster were reported. An entropic measurement based on the probability of an occupied site belonging to an $s$-site cluster was derived, showing that the maximum entropy is attained at the percolation threshold. We demonstrated that in an ideal situation the maximum of cluster size diversity and the percolation threshold for the $1 d$ case are the same. Moreover, similarities in the scaling of $p_{c}\left(D_{\max }\right)$ and the probability of the percolation threshold $p_{c}$ versus the lattice dimension, give further evi- 
dence for the conjecture that the two probabilities, also for the higher lattice dimension, are indeed the same.

The statistics of cluster size diversity show some interesting implications for a possible statistical description of the Universe as a complex system, since we live in a diversified Universe, which produces structures spanning all possible length scales. Following the arguments of $[14,15]$, we presented further evidence for the sustainability of complex systems for $d=3$, which lies in a compromise between the ca- pacity of the system to support a diversified structure and the energy necessary to attain this complex state.

\section{ACKNOWLEDGMENTS}

We would like to acknowledge M.A.F. Gomes for insightful discussions at the early stage of this work and F. Van Rossen for carefully reading the manuscript. This work was supported by CAPES (Brazilian Government Agency).
[1] D.M. Thompson, On Growth and Form (Cambridge University Press, Cambridge, 1971).

[2] D.M. Raup, S.J. Gould, T.J.M. Schopf, and D.S. Simberloff, J. Geol. 81, 525 (1973).

[3] S.A. Kauffman, The Origins of Order (Oxford University Press, Oxford, 1993).

[4] S. Wolfram, Theory and Applications of Cellular Automata (World Scientific, Singapore, 1986).

[5] S. Wolfram, Cellular Automata and Complexity: Collected Papers (Addison-Wesley, Reading, MA, 1994).

[6] B.B. Mandelbrot, The Fractal Geometry of Nature (Freeman, New York, 1983).

[7] H.O. Peitgen and P. H. Richter, The Beauty of Fractals: Images of Complex Dynamical Systems (Springer-Verlag, Berlin, 1986).

[8] K. Coutinho, M.A.F. Gomes, and S.K. Adhikari, Europhys. Lett. 18, 119 (1992).

[9] P. Meakin, Fractals, Scaling and Growth Far from Equilibrium (Cambridge University Press, Cambridge, 1998).

[10] B.A. Huberman and T. Hogg, Physica D 22, 376 (1986).

[11] J.P. Crutchfield, Physica D 75, 11 (1994).

[12] R. Badii and A. Politi, Complexity: Hierarchical Structures and Scaling in Physics (Cambridge University Press, Cambridge, 1997).
[13] P. Grassberger, Helv. Phys. Acta 62, 489 (1989).

[14] M.A.F. Gomes, F.A.O. Souza, and S.K. Adhikari, J. Phys. A 28, L613 (1995).

[15] J.B.C. Garcia, M.A.F. Gomes, T.I. Jyh, T.I. Ren, and T.R.M. Sales, in The Evolution of Complexity, edited by F. Heylighen (Kluwer, Dordrecht, in press).

[16] J.B.C. Garcia, M.A.F. Gomes, T.I. Jyh, T.I. Ren, and T.R.M. Sales, Phys. Rev. E 48, 3345 (1993).

[17] T.R.M. Sales, J.B.C. Garcia, T.I. Jyh, T.I. Ren, and M.A.F. Gomes, Physica A 197, 604 (1993).

[18] T.R.M Sales, Phys. Rev. E 48, 2118 (1993).

[19] K.R. Coutinho, M.D. Coutinho-Filho, M.A.F. Gomes, and A.M. Nemirovsky, Phys. Rev. Lett. 72, 37 (1994).

[20] I.R. Tsang and I.J. Tsang, J. Phys. A 30, L239 (1997).

[21] I.J. Tsang and I.R. Tsang, in Unifying Themes in Complex Systems: Proceedings of the First Necsi International Conference on Complex Systems, edited by Yaneer Bar-Yam (Perseus Book, Reading, MA, 1999), also available at InterJournal http://www.interjounal.org/.

[22] I.J. Tsang, I.R. Tsang, and D. Van Dyck (unpublished).

[23] J. Hoshen and R. Kopelman, Phys. Rev. E 14, 3438 (1976).

[24] D. Stauffer and A. Aharony, Introduction to Percolation Theory, revised 2nd ed. (Taylor \& Francis, London, 1994).

[25] H.A. Simon, Proc. Am. Philos. Soc. 106, 467 (1962). 